\title{
Reproducibility of range of motion and muscle strength measurements in patients with hip osteoarthritis - an inter-rater study
}

Erik Poulsen ${ }^{1,2^{*}}$, Henrik Wulff Christensen ${ }^{2}$, Jeannette Østergaard Penny ${ }^{3,4}$, Søren Overgaard ${ }^{3,4}$, Werner Vach ${ }^{2,5}$ and Jan Hartvigsen ${ }^{1,2}$

\begin{abstract}
Background: Assessment of range of motion (ROM) and muscle strength is fundamental in the clinical diagnosis of hip osteoarthritis (OA) but reproducibility of these measurements has mostly involved clinicians from secondary care and has rarely reported agreement parameters. Therefore, the primary objective of the study was to determine the inter-rater reproducibility of ROM and muscle strength measurements. Furthermore, the reliability of the overall assessment of clinical hip OA was evaluated. Reporting is in accordance with proposed guidelines for the reporting of reliability and agreement studies (GRRAS).
\end{abstract}

Methods: In a university hospital, four blinded raters independently examined patients with unilateral hip OA; two hospital orthopaedists independently examined 48 (24 men) patients and two primary care chiropractors examined 61 patients (29 men). ROM was measured in degrees (deg.) with a standard two-arm goniometer and muscle strength in Newton (N) using a hand-held dynamometer. Reproducibility is reported as agreement and reliability between paired raters of the same profession. Agreement is reported as limits of agreement (LoA) and reliability is reported with intraclass correlation coefficients (ICC). Reliability of the overall assessment of clinical OA is reported as weighted kappa.

Results: Between orthopaedists, agreement for ROM ranged from LoA [-28-12 deg.] for internal rotation to [-8-13 deg.] for extension. ICC ranged between 0.53 and 0.73 , highest for flexion. For muscle strength between orthopaedists, LoA ranged from [-65-47N] for external rotation to [-10 $-59 \mathrm{~N}]$ for flexion. ICC ranged between 0.52 and 0.85 , highest for abduction. Between chiropractors, agreement for ROM ranged from LoA [-25-30 deg.] for internal rotation to [-13-21 deg.] for flexion. ICC ranged between 0.14 and 0.79 , highest for flexion. For muscle strength between chiropractors, LoA ranged between [-80-20N] for external rotation to [-146-55N] for abduction. ICC ranged between 0.38 and 0.81, highest for flexion. Weighted kappa for the overall assessment of clinical hip OA was 0.52 between orthopaedists and 0.65 between chiropractors.

Conclusions: Reproducibility of goniometric and dynamometric measurements of ROM and muscle strength in patients with hip OA is poor between experienced orthopaedists and between experienced chiropractors.

Orthopaedists and chiropractors can to a moderate degree differentiate between hips with or without osteoarthritis.

Keywords: Hip, Examination, Inter-observer, Reliability, Osteoarthritis, Hip

\footnotetext{
* Correspondence: e.poulsen@nikkb.dk

'Institute of Sports Science and Clinical Biomechanics, University of Southern Denmark, Odense, Denmark

${ }^{2}$ Nordic Institute of Chiropractic and Clinical Biomechanics, Odense, Denmark

Full list of author information is available at the end of the article
}

\section{Biomed Central}

(c) 2012 Poulsen et al.; licensee BioMed Central Ltd. This is an Open Access article distributed under the terms of the Creative Commons Attribution License (http://creativecommons.org/licenses/by/2.0), which permits unrestricted use, distribution, and reproduction in any medium, provided the original work is properly cited. 


\section{Background}

In primary care, when patients over 40 years of age present with hip pain, the most common diagnosis is osteoarthritis (OA) [1,2]. A combination of radiographic signs and clinical findings is usually recommended for confirming the diagnosis. But although approximately half demonstrate definite radiological signs of OA [1], radiographs are not recommended solely for just confirming the diagnosis. Thus, the clinical exam is of key importance [3]. Clinical practice guidelines recommend assessment of range of motion (ROM) and muscle strength when adult patients present with hip pain [4] and the two clinical signs documented to correlate with hip OA besides pain are reduced ROM [5-8] and muscle strength [5,8-11]. Reduced ROM is further documented as a clinical predictor for hip OA $[2,12]$ and in patients with mild symptomatic hip OA, specific ranges of reduced ROM are correlated with radiographic signs [13].

A number of studies have evaluated the reliability of ROM and muscle strength measurements in patients with hip OA and reported moderate to excellent reliability $[6,7,14-19]$. But the presence of methodological issues raises questions about the external validity of these results. Equipment ill-suited for clinical practice has been used $[7,18]$ or the number of study subjects has been small, limiting the between-subject variation $[6,14,16,17]$. Inappropriate correlation coefficients have been reported $[14,15]$ or reliability coefficients have been reported alone, ignoring agreement parameters $[15,17,19]$. Reliability coefficients indicate the procedure's ability to discriminate between patients, whereas agreement parameters reflect error between repeated measurements $[16,17]$. So, when measurements are used to assess change over time, agreement parameters should be reported [20].

Intra-rater reproducibility is commonly found to be more reliable than inter-rater reproducibility because between-rater variability is eliminated [21-23]. In clinical or research settings, intra-rater reproducibility could be adequate where only one rater performs the measurements, whereas inter-rater reproducibility is essential for clinicians when follow-up consultations on the same patient are performed by different clinicians or when clinicians have to agree on a diagnosis. Three studies have examined inter-rater reliability of ROM measurements on hip OA patients but none reported agreement parameters $[16,17,24]$. One study reported inter-rater reliability on muscle strength measurements in hip OA patients but agreement parameters were not reported [17]. Only one study evaluating reproducibility among primary care clinicians has been identified [16].

Therefore, the primary purpose of this study was to assess the inter-rater reproducibility of passive ROM and muscle strength measurements in patients with unilateral hip OA among clinicians in both primary care and hospital secondary care. The secondary purpose was to assess the inter-rater reliability of the degree of clinical hip OA among the same clinicians based on findings of ROM and strength measurements.

\section{Methods \\ Participants}

The study participants took part in a randomised clinical trial described elsewhere [25]. Recruitment of the participants is illustrated in Figure 1. Inclusion criteria included unilateral hip pain $>3$ months and unilateral radiographic hip OA on the painful side. The complete lists of inclusion and exclusion criteria are presented in Table 1. Prior to examination, each participant completed a questionnaire with details on age, gender, height, weight, side of hip pain, duration of complaint and pain severity. The participant reported average pain experienced during the previous week and worst pain experienced during the previous week.

Prior to their involvement, each participant received verbal and written information about the study and signed a written consent form. The study was granted approval by the Regional Ethics Committee of Southern Denmark, approval number S-20080027 and was registered and approved by the Danish Data Protection Agency, J.nr. 2008-41-1910.

\section{Raters}

Four raters participated. There were two medical doctors from hospital care: one male senior orthopaedic surgeon specialising in hip surgery with clinical experience of $>20$ years and one female first year resident in orthopaedic surgery with 4 years' experience. And there were two male chiropractors working in primary care, both with clinical experience of $>20$ years: one with 8 years of clinical interest in specific hip conditions and one with no specific interest or clinical experience with hip conditions. At the time of examination, these raters were aware of the inclusion- and exclusion criteria but had no prior knowledge of which side of the body involved the hip condition and they were blind to the radiographic findings.

\section{Setting and equipment}

All examinations took place at Odense University Hospital, Denmark. Passive hip ROM was measured using a standard two-arm plastic goniometer, $30 \mathrm{~cm}, 0-360$ degrees (deg.) with single deg. increments (MSD Europe bvba). Recordings were made to the nearest five deg. Hip muscle strength was measured in Newtons (N) using a hand-held dynamometer (HHD), model MicroFet II (Hoggan Health Industries Inc.). The goniometer and HHD were chosen as they are inexpensive and easy to implement in both primary and outpatient hospital 


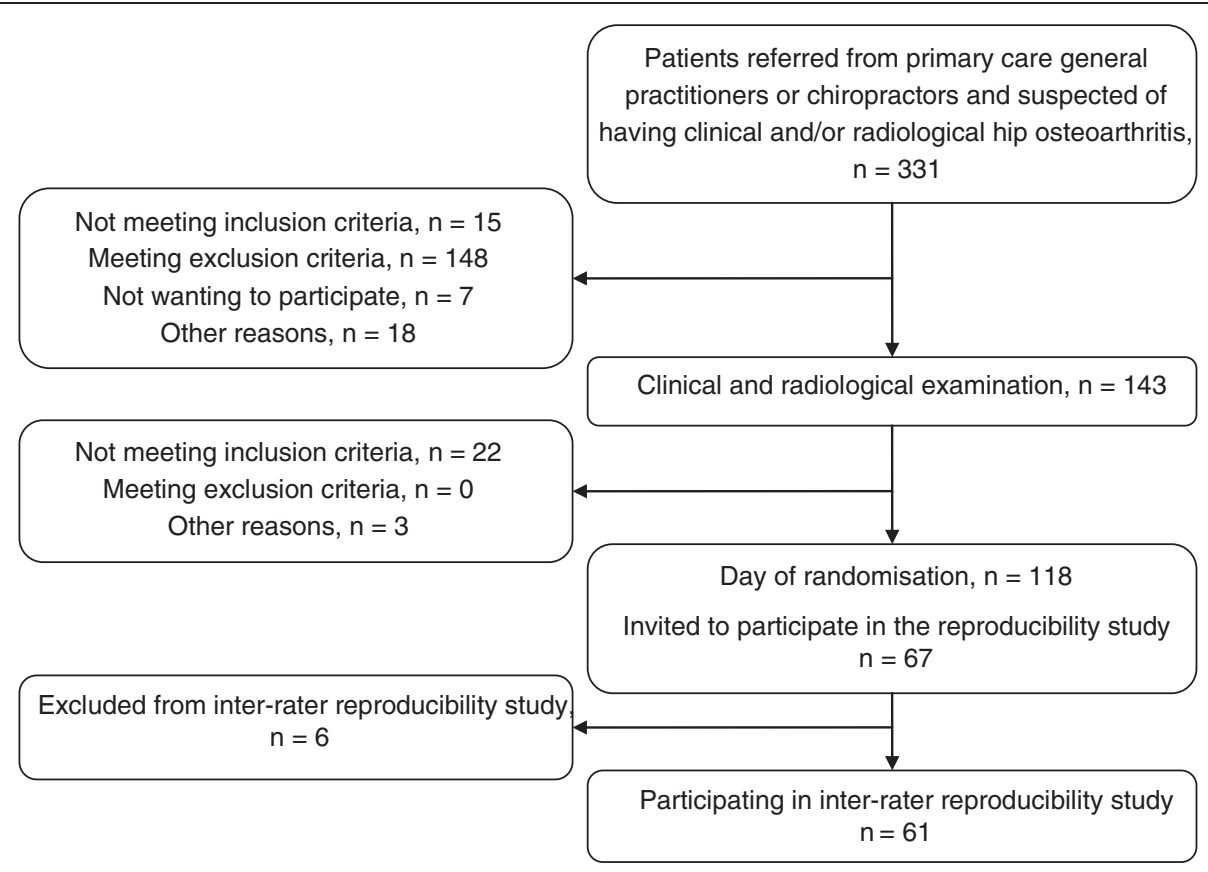

Figure 1 Flow chart of participants included in the study.

care. It was decided to test them on raters with minimal protocol standardisation and without rigorous training.

\section{Procedures}

The protocol for the examination procedures is attached as an appendix [see Additional file 1]. The aim of the protocol was to resemble test procedures used in daily practice and it was created by consensus between the raters.

A day was scheduled to familiarise raters with the use of the equipment and rehearse individual examination procedures. Two university students acted as study

\section{Table 1 Inclusion and exclusion criteria for participants}

\begin{tabular}{ll}
\hline Inclusion criteria & Exclusion criteria \\
\hline$-40-80$ years of age & - Bilateral hip pain \\
- Unilateral hip pain $>3$ months & - Previous hip or knee joint replacement surgery \\
- Radiographic hip osteoarthritis $(\mathrm{OA})$ defined by joint space & - Indication for hip joint replacement surgery within the next 6 months \\
width measurements $<2.00 \mathrm{~mm}$ or a side difference $>10 \%$ & \\
- Ability to speak and read Danish & - Hip OA due to hip fracture or infection \\
& - Rating of worst hip pain during the last week as $\leq 2$ on an 11 -box numerical \\
& rating scale \\
& - Manual therapy for the hip within the last 12 months \\
& - Hip dysplasia; Center Edge angle $<25$ degrees and Acetabular index Angle $>10$ \\
& degrees \\
& - Local knee pain originating from the knee on the same side as the hip OA \\
& - Low back pain dominating over hip symptoms \\
& - Inflammatory joint disease \\
& - Polyarthritis defined as $>3$ regional sites \\
& - Cerebrovascular disease \\
& - Polyneuropathy or neuromuscular disease \\
& - Malignant disease \\
& - Conditions other than hip OA appearing to be the cause of the \\
& patient's symptoms \\
& - Refusal to participate \\
&
\end{tabular}


subjects. Initially, measurements for ROM and strength were included for all six directions of movement, i.e. extension, flexion, abduction, adduction, internal and external rotation. Strength testing in adduction was excluded due to consensus on issues concerning practicality and interpretation when examining this patient group. The procedure requires stability of the pelvis and opposite leg during testing and HHD placement includes lower leg strength. In order to detect differences in maximum strength in patients with early to mild hip osteoarthritis, it was decided to use a break test and not an isometric test [26]. The protocol was revised and a training day was scheduled with eight patients with hip pain and radiographic hip OA. Following the training session, corrections were made regarding the positioning of participants. The final protocol was approved by all raters. Measurements were performed on both hips.

On the days of data collection, four separate cubicles were created by room dividers with identical examination tables. Four participants were asked to each enter a cubicle, undress to their underwear and wait for a rater. Each participant was then examined by the four raters in turn, randomly rearranging the sequence of raters after each examination to minimise any possible learning effect. Raters were free to determine which hip to examine first. Communication between rater and participants regarding examination procedures was allowed but information pertaining to the participant's case history was not. No communication between raters was allowed in between sessions. An assistant was assigned to each rater to record the result of the examination findings on a standardised form and to assist holding the goniometer during ROM in extension. ROM was measured once and muscle strength measured twice.

Following completion of all measurements, each rater independently assessed each hip for the degree of clinical hip OA and assigned it to one of three categories: no hip OA, mild hip OA or severe hip OA. The decision of the category was based on the opinion of each rater.

For generalisability and to obtain a representative study sample it was decided to include a minimum of 60 participants.

\section{Statistical analysis}

Double data entry was performed by a person not involved in the study. Descriptive statistics are presented for participant characteristics. For the continuous variables of hip ROM and muscle strength, means and standard deviations (SDs) for each rater are reported, and since we were interested in the reproducibility between raters of the same profession, i.e. orthopaedists and chiropractors, pair-wise mean differences and SDs between raters of the same profession are reported. The value reported for muscle strength is an average of two measurements. Bland and Altman plots were inspected visually for indication of heteroscedasticity. Measurement error is reported as standard error of the measurement $\left(\mathrm{SEM}_{\text {agreement }}\right)$ described by de Vet et al. and is reported for the purpose of comparison with other studies [20]. SEM agreement incorporates measurement error between raters and error from interaction between raters and participants.

Agreement between raters is reported as 95\% limits of agreement (LoA) as described by Bland and Altman where the clinical interpretation is based on the $95 \%$ range [27]. So, if the systematic rater error between two raters is zero, half the range can be considered the smallest detectable change (within 95\% confidence). Percent agreements between raters are reported for ROM as agreement within $10 \mathrm{deg}$. for flexion and $5 \mathrm{deg}$. for all other ROMs. Ten deg. for flexion was chosen since the range in flexion is considerably larger. Clinically acceptable percent agreement between clinicians was set a priori to $75 \%$. Reliability is reported with the intraclass correlation coefficient $\left(\mathrm{ICC}_{2.1}\right)$ including $95 \%$ confidence intervals and is reported within raters of the same profession. Interpretation of ICC is according to the classification: < 0.69, poor; 0.70-0.79, fair; 0.80-0.89, good; 0.90-1.00, excellent [28]. Acceptable reliability was set a priori at $\geq 0.70$ [29]. $\mathrm{ICC}_{2.1}$ was used in order to generalise the to a wider population of raters [30]. The reliability of the overall assessment of clinical hip OA is reported with Cohen's weighted kappa. The interpretation of Cohen's weighted kappa is according to the classification by Landis and Koch [31]: <0.00, poor; 0.00-0.20, slight; 0.21-0.40, fair; 0.41-0.60, moderate; 0.61-0.80, substantial, 0.81-1.00, almost perfect. Kappa is weighted as $1.0 / 0.5 / 0.0$. Acceptable kappa values were set a priori at $\geq 0.60$. Analysis was performed using Stata 10 software (StataCorp, Texas, USA).

\section{Results}

Sixty-seven participants were invited to take part in the study. Three were excluded due to bilateral hip pain, one due to neuropathy, one for having no radiographic signs of hip OA and one failed to attend, resulting in 61 participants. Inclusion of participants took place from January 2009 to September 2009 and a total of 5 days evenly distributed throughout the period were used for examinations. The senior orthopaedic surgeon was not available for one of these days, so a total of 48 participants were assessed for comparison between the two orthopaedists. Results are only presented for the hip with clinical and radiographic OA. Descriptive participant characteristics are listed in Table 2. Means and SDs for ROM and strength measurements for all four raters are listed in Table 3 as well as pair-wise mean differences and SDs between orthopaedists and between chiropractors. 


\section{Table 2 Characteristics of participants}

\begin{tabular}{ll}
\hline Involved side, right / left ( $\mathrm{n})$ & $36 / 25$ \\
Gender, men / women ( $\mathrm{n})$ & $29 / 32$ \\
Mean age in years (SD) & $65.6(8.0)$ \\
Mean body mass index in $\mathrm{kg} / \mathrm{m}^{2}$ (SD) & $26.8(3.4)$ \\
Mean duration of symptoms in months (SD) & $37(32)$ \\
Mean average level of pain during the last week & $(\mathrm{SD})$ \\
$\begin{array}{l}\text { Mean worst level of experienced pain during the last } \\
\text { week* (SD) }\end{array}$ & $4.7(1.8)$ \\
\hline
\end{tabular}

$\mathrm{N}$, numbers; SD, standard deviation. *Scores rated on a 0-10 Likert scale, $0=$ no pain, $10=$ worst possible pain.

$\mathrm{SEM}_{\text {agreement, }}$ percent agreement for ROM, LoA and ICC for the pair-wise comparison are also listed in Table 3.

Statistically significant differences $(\mathrm{p}<0.05)$ were found in general between all pair-wise measurements. But specific patterns for ROM measurements were not noted for the pair-wise comparisons. One chiropractor demonstrated systematically higher values for all hip muscle strength measurements. The systematic difference for the individual measurements is further reflected in the LoA with the upper and lower limits deviating nonsymmetrically from zero. Visual inspection of the Bland and Altman plots did not indicate heteroscedasticity.

Percent agreement for ROM between orthopaedists ranged between 42 and 79\%. Between chiropractors, the range was $31-83 \%$. Between orthopaedists, LoA for ROM ranged from [-8-13 deg.] for extension to [-28-11 deg.] for internal rotation and between chiropractors the range was from [-13-21 deg.] for flexion to [-25-30 deg.] for internal rotation. LoA for internal rotation between orthopaedists are illustrated in Figure 2 and between chiropractors in Figure 3. Reliability for ROM between orthopaedists ranged from 0.53 (95\% CI 0.26-0.72) for external rotation to 0.73 (0.38-0.87) for flexion. Between chiropractors, the range was 0.14 $(-0.09-0.36)$ for internal rotation to $0.79(0.63-0.88)$ for flexion.

For muscle strength, LoA between orthopaedists ranged from [-65-47N] for external rotation to [-101-59N] for flexion and between chiropractors, the range was from $[-80-20 \mathrm{~N}]$ for external rotation to $[-146-55 \mathrm{~N}]$ for abduction. LoA for abduction between orthopaedists are illustrated in Figure 4 and between chiropractors in Figure 5. ICC for orthopaedists ranged from 0.52 (0.29-0.70) for internal rotation to 0.85 (0.29-0.70) for abduction. For chiropractors, the ICC ranged from 0.38 (0.00-0.64) for abduction to 0.81 (0.69-0.88) for flexion.

Between orthopaedists, reliability (weighted kappa) for the degree of clinical hip OA based on ROM and muscle strength assessment was 0.52 and between chiropractors, 0.65 .

\section{Discussion}

To our knowledge, this is the first study involving clinicians from both primary care (chiropractors) and hospital secondary care (orthopaedists). We found generally poor to moderate inter-rater reproducibility for all ROM and muscle strength measurements both between orthopaedists and between chiropractors. Acceptable reproducibility was found only for hip ROM in flexion, both between orthopaedists and between chiropractors. Reliability for the assessment of clinical hip OA is moderate both between orthopaedists and between chiropractors.

\section{Clinical interpretation}

When incorporating the measurement error into a clinical context, the wide limits of all LoAs for ROM for both orthopaedists and chiropractors indicate that an effect following intervention should be a minimum of 17 deg. for flexion, 10 for extension, 15 for abduction, 12 for adduction and 20 for internal rotation and 17 for external rotation before it with ( $95 \%$ confidence) can be distinguished from random fluctuations due to measurement error, if measured by two different raters. Considering the normal range for flexion and abduction, this is possible but unlikely for extension, adduction and internal and external rotation. Interpretation of the results for flexion and abduction though must be done with care as Müller and Büttner argue the ICC is "dependent on the range of the measuring scale" [32]. So the larger the scale, the higher the coefficient and the range for flexion and abduction is considerably larger than the other ROMs of the hip. The clinical interpretation of reliability must involve the lower 95\% CIs which further reflect the poor to moderate findings [33]. Only muscle strength for abduction between orthopaedists demonstrated acceptable lower 95\% CI of 0.74 and between chiropractors for flexion with lower $95 \% \mathrm{CI}$ of 0.69 .

For hip muscle strength, the same interpretation of LoA is not possible as muscle strength diminishes with each decade and is up to $50 \%$ higher in males [26]. Further, variation in force applied between raters can be significantly different and between raters of opposite sex $[34,35]$. The latter was not apparent between the orthopaedists as mean flexion and external rotation was significantly higher for the female orthopaedist.

Observing the results between the two orthopaedists and the two chiropractors did not give any indication of one group of professionals producing more reliable measurements than the other. However, the reliability measures between chiropractors were lower when assessing both ROM and muscle strength and could reflect that their clinical practice clientele are typically not solely hip pain patients. The variation between the orthopaedic surgeon and the first year intern probably reflects the difference in experience. 
Table 3 Inter-rater reproducibility of hip range of motion (deg.) and muscle strength (N) for 2 orthopaedists and 2 chiropractors

\begin{tabular}{|c|c|c|c|c|c|c|c|}
\hline $\begin{array}{l}\text { Hip range of motion } \\
\text { measured with a } \\
\text { goniometer to the } \\
\text { nearest } 5 \text { deg. }\end{array}$ & $\begin{array}{l}\text { Ortho } 1 \text { (deg.) } \\
\text { Mean (SD) }\end{array}$ & $\begin{array}{l}\text { Ortho } 2 \text { (deg.) } \\
\text { Mean (SD) }\end{array}$ & $\begin{array}{l}\text { Mean } \\
\text { difference } \\
\text { (SD) }\end{array}$ & $\begin{array}{l}\text { Percent } \\
\text { agreement } \\
(\%)^{*}\end{array}$ & $\begin{array}{l}\text { LOA } \\
\text { (deg.) }\end{array}$ & SEM† & $\begin{array}{l}\text { ICC } \\
(95 \% \mathrm{CI})\end{array}$ \\
\hline Flexion $(n=48)$ & $113(18)$ & $106(14)$ & $7(10) \neq$ & 71 & $-12-26$ & 8 & $0.73(0.38-0.87)$ \\
\hline Extension $(n=48)$ & $6(6)$ & $4(8)$ & $2(5) \neq$ & 79 & $-8-13$ & 4 & $0.68(0.46-0.81)$ \\
\hline Abduction $(n=48)$ & $29(10)$ & $26(8)$ & $3(7) \neq$ & 67 & $-12-17$ & 6 & $0.63(0.41-0.76)$ \\
\hline Adduction $(\mathrm{n}=48$ ) & $17(7)$ & $17(7)$ & $0(6)$ & 77 & $-11-12$ & 4 & $0.65(0.45-0.79)$ \\
\hline $\begin{array}{l}\text { Internal rotation } \\
(n=48)\end{array}$ & $4(14)$ & $12(14)$ & $-8(10) \neq$ & 42 & $-28-12$ & 9 & $0.63(0.17-0.82)$ \\
\hline External rotation & $28(11)$ & $33(7)$ & $-4(9) \neq$ & 69 & $-21-13$ & 6 & $0.53(0.26-0.72)$ \\
\hline
\end{tabular}

$(n=48)$

\begin{tabular}{|c|c|c|c|c|c|c|c|}
\hline $\begin{array}{l}\text { Hip muscle strength } \\
\text { measured with a } \\
\text { dynamometer in } \\
\text { Newton (N) }\end{array}$ & Strength $(\mathrm{N})$ & Strength (N) & & & (N) & & \\
\hline Abduction $(n=48)$ & $213(65)$ & $212(67)$ & $0(37)$ & & $-72-72$ & 26 & $0.85(0.74-0.91)$ \\
\hline Flexion $(n=48)$ & $167(42)$ & $185(50)$ & $-21(41) \neq$ & & $-101-59$ & 32 & $0.55(0.28-0.73)$ \\
\hline Internal rotation $(n=48)$ & $151(38)$ & $146(36)$ & $5(36)$ & & $-66-76$ & 26 & $0.52(0.29-0.70)$ \\
\hline External rotation $(n=48)$ & $123(34)$ & $132(39)$ & $-9(28) \neq$ & & $-65-47$ & 21 & $0.68(0.49-0.81)$ \\
\hline $\begin{array}{l}\text { Hip range of motion } \\
\text { measured with a } \\
\text { goniometer to the } \\
\text { nearest } 5 \text { deg. }\end{array}$ & $\begin{array}{l}\text { Chiro } 1 \text { (deg.) } \\
\text { Mean (SD) }\end{array}$ & $\begin{array}{l}\text { Chiro } 2 \text { (deg.) } \\
\text { Mean (SD) }\end{array}$ & $\begin{array}{l}\text { Mean } \\
\text { difference } \\
\text { (SD) }\end{array}$ & $\begin{array}{l}\text { Percent } \\
\text { agreement } \\
(\%)^{*}\end{array}$ & LOA (deg.) & SEM+ & ICC (95\% CI) \\
\hline Flexion $(n=61)$ & $112(13)$ & $108(15)$ & 4 (9)† & 83 & $-14-21$ & 7 & $0.79(0.63-0.88)$ \\
\hline Extension $(n=61$ ) & $9(5)$ & $14(5)$ & $-6(5) \neq$ & 66 & $-16-5$ & 5 & $0.33(0.06-0.61)$ \\
\hline Abduction $(n=61)$ & $29(9)$ & $22(9)$ & $7(8) \neq$ & 46 & $-8-23$ & 8 & $0.45(0.01-0.71)$ \\
\hline Adduction $(n=61)$ & $17(7)$ & $14(5)$ & $3(8) \neq$ & 61 & $-13-19$ & 6 & $0.14(0.09-0.36)$ \\
\hline $\begin{array}{l}\text { Internal rotation } \\
(n=61)\end{array}$ & $13(10)$ & $11(16)$ & $2(14)$ & 31 & $-25-30$ & 10 & $0.44(0.21-0.62)$ \\
\hline $\begin{array}{l}\text { External rotation } \\
(n=61)\end{array}$ & $32(9)$ & $30(12)$ & $2(11)$ & 59 & $-19-23$ & 8 & $0.48(0.27-0.65)$ \\
\hline $\begin{array}{l}\text { Hip muscle strength } \\
\text { measured with } \\
\text { a dynamometer in } \\
\text { Newton (N) }\end{array}$ & Strength $(\mathrm{N})$ & Strength (N) & & & (N) & & \\
\hline Abduction $(n=61)$ & $141(42)$ & $186(61)$ & $-45(51) \neq$ & & $-146-55$ & 48 & $0.38(0.00-0.64)$ \\
\hline Flexion $(n=61)$ & $165(50)$ & $178(65)$ & $-11(35) \neq$ & & $-79-56$ & 26 & $0.81(0.69-0.88)$ \\
\hline $\begin{array}{l}\text { Internal rotation } \\
(n=61)\end{array}$ & $142(43)$ & $190(49)$ & $-48(34) \neq$ & & $-115-19$ & 42 & $0.48(0.09-0.77)$ \\
\hline $\begin{array}{l}\text { External rotation } \\
(\mathrm{n}=61)\end{array}$ & $120(34)$ & $150(48)$ & $-30(26) \neq$ & & $-80-20$ & 28 & $0.65(0.00-0.82)$ \\
\hline
\end{tabular}

The level of standardisation and minimal training is likely to have influenced the systematic differences seen in almost all individual measurements. As differences were not systematically higher for one specific rater across individual ROMs, individual habits such as placement of the instrument and rater's force are likely to be the cause. The poor results of ROM in internal and external rotation could reflect participants being positioned supine and not sitting, as position is known to influence the precision of individual measurements [17]. One chiropractor 


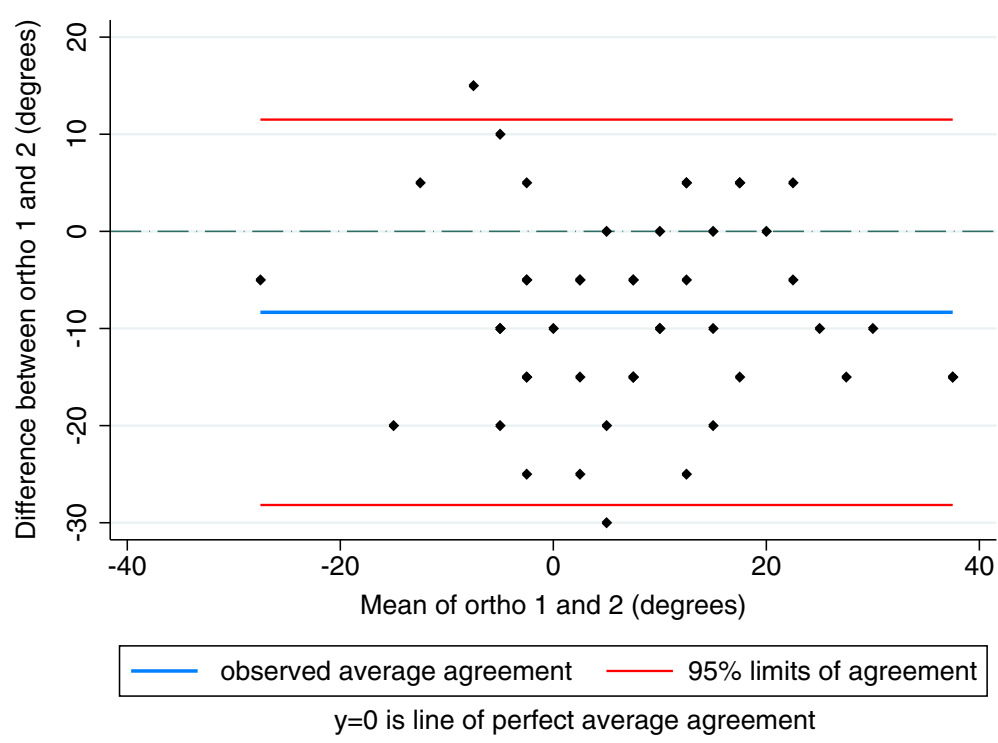

Figure 2 Limits of agreement between two orthopaedists for hip internal rotation range of motion (degrees).

had higher measurements for all strength tests, which is likely to be attributed to the force generated during the break test and in inter-rater variability interpreting when the break test is accomplished. The recorded variation in muscle strength could be due to fatigue from repetitive testing as participants were examined four times. We consider this effect minimal, as examinations were scheduled with a 15-minute interval and each session of strength testing lasted no more than 5 minutes. This allowed time for the ROM examination, a resting period for the participant and a change-over of raters. The results are also likely to be influenced by the orthopaedists or chiropractors having limited experience with the
HHD. The procedures were tested in a validation study as part of the randomised clinical trial mentioned earlier (data not published). The rater tested had similar experience with the HHD and demonstrated similar levels of intra-rater reliability but with much narrower LoA intervals. For ROM measurements, the rater demonstrated clinically acceptable intra-rater reproducibility without routine use of a goniometer in practice.

\section{Comparison with other studies}

Several studies have documented from poor to excellent inter-rater reliability of ROM in patients with hip OA using a goniometer. Sutlive et al. found fair to good

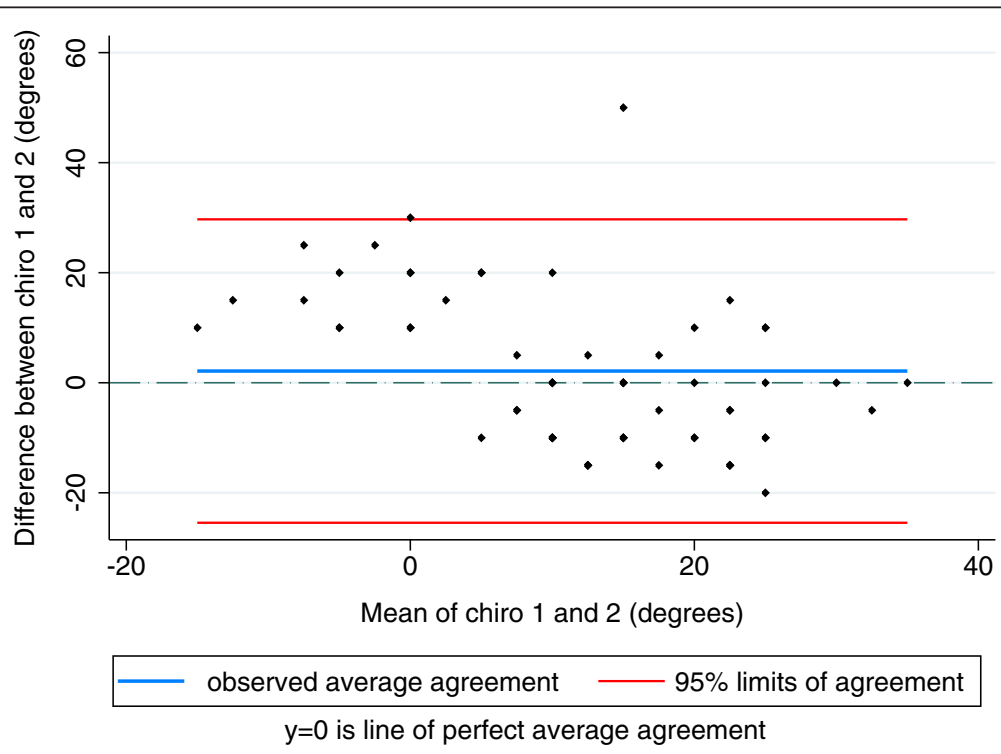

Figure 3 Limits of agreement between two chiropractors for hip internal rotation range of motion (degrees). 


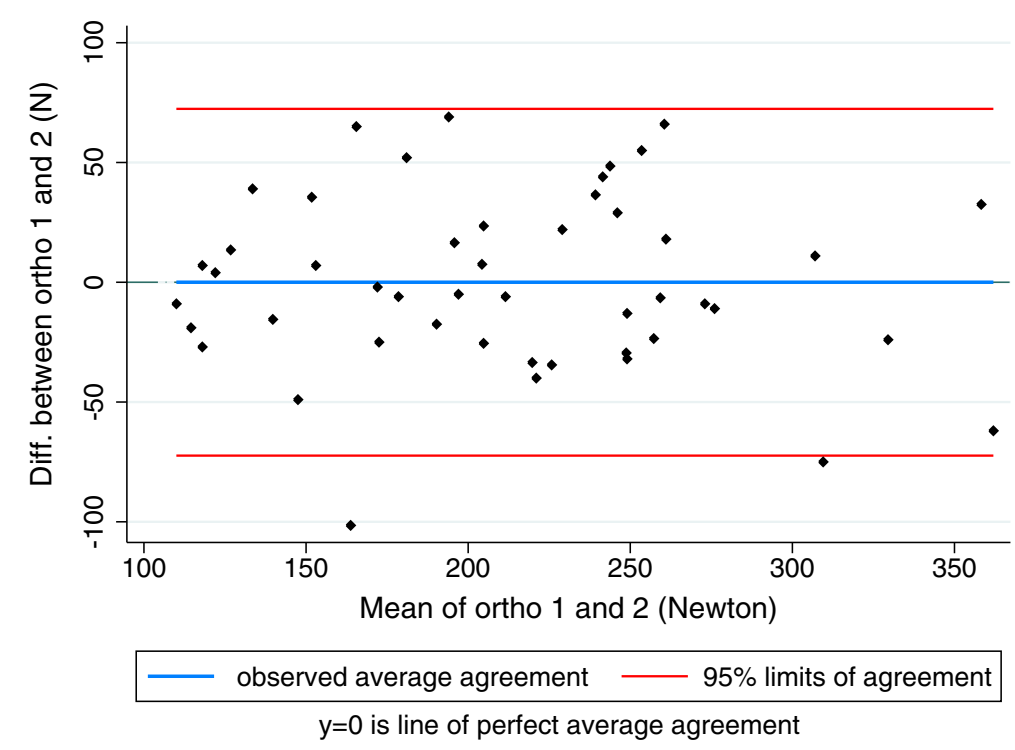

Figure 4 Limits of agreement between two orthopaedists for abduction hip strength (Newton).

reliability but agreement parameters were not reported [19]. Holm et al. studied teams of raters but results for mean measurements of each ROM were combined from all raters [14]. Cibere et al. found clinically acceptable reliability both before and after standardisation of ROM and muscle strength measurements but they did not incorporate variance components from the patients or random error and agreement parameters were not reported [17]. Theiler et al. reported reliability coefficients similar to those in our study but used Pearson's correlation coefficient which does not incorporate systematic differences between raters [15]. For hip muscle strength, Arnold et al. found excellent inter-rater reliability using a different HHD model but subjects were a mix of patients with both hip and knee OA [36]. Studies have documented good to excellent intra- and interrater reliability on healthy subjects using goniometer and HHD but they are not comparable to hip OA patients as age and disease characteristics influence the variation between subjects [21-23,35,37,38].

\section{Study limitations}

There are a number of limitations associated with this study. First, raters were aware of the participant'

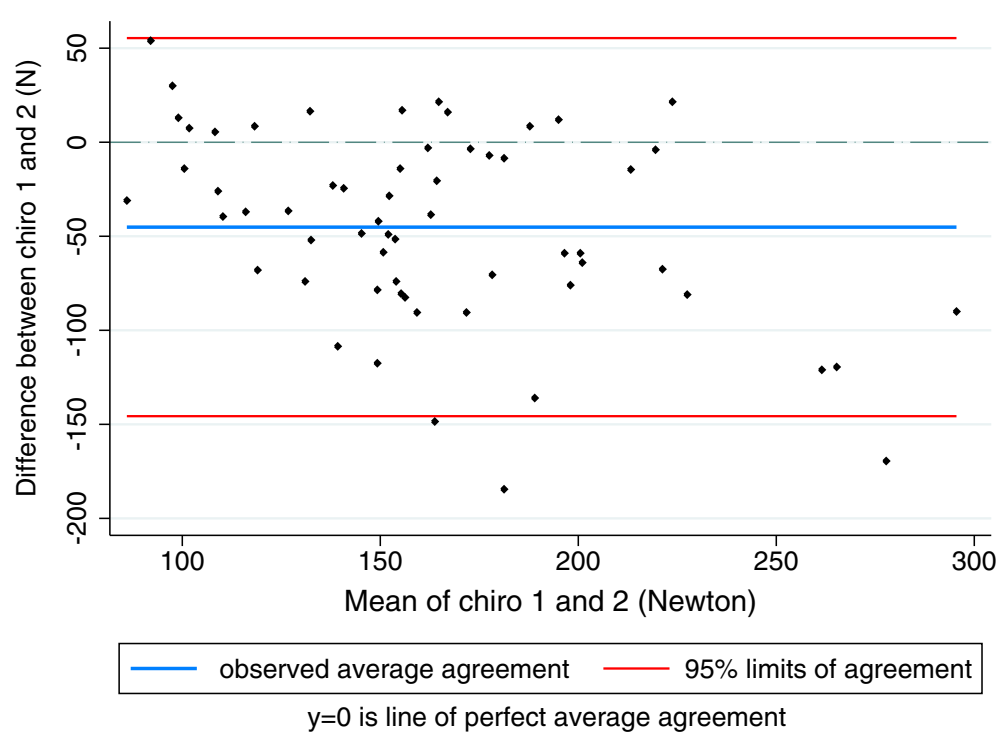

Figure 5 Limits of agreement between two chiropractors for abduction hip strength (Newton). 
inclusion criterion of unilateral clinical and radiographic hip OA, so in the context of the clinical setting, no other hip conditions had to be considered. Second, the study did not involve rigorous training of the raters; however, we were interested in results reflecting current clinical practice. Several studies have reported on the added effect of protocol standardisation and rigorous training in musculoskeletal medicine $[17,39,40]$ and such training could potentially result in better agreement. Third, the raters had prior knowledge of patients having unilateral clinical and radiographic hip OA which could inflate reliability coefficients. When one hip was examined, the rater would know if the other hip would be affected by OA or not. Fourth, the orthopaedic surgeon was not available for one of the examination sessions, so only 48 participants were included in the analysis between orthopaedists, instead of the 61 originally recruited. Fifth, the assessment of clinical hip OA was based solely on ROM and muscle strength evaluation. In clinical practice, a more extensive list of individual tests is used as well as information from the patient's case history. It is further possible that the overall assessment was influenced by indications of a procedure being painful, to which the raters were not blinded. Sixth, we decided to omit adductor strength testing even though adductor strength has been documented to be reduced in patients with hip OA [5,9]. But measurement equipment has not been suitable for the clinical setting and in this patient group we concluded on the training day that stability of the pelvis and opposite leg were insufficient. We are aware that reproducibility of adductor strength testing by HHD on young healthy subjects has been reported as clinically acceptable [41]. Last, differentiation between levels of clinical hip OA following the overall assessment was only made from mild to severe hip OA. In the assessment of radiographic hip OA, it is common to categorise into none, mild, moderate and severe.

The literature on reproducibility of the clinical hip examination in patients with hip OA is limited and heterogeneous but recently the first set of guidelines on the reporting of reliability and agreement studies was published [33]. As patient characteristics differ in symptom and disease severity in primary and hospital care, future studies should take place in the setting where patient populations are examined and managed and involve clinicians from the same setting. To improve external validity, more than two clinicians should be included and selected randomly from an appropriate population of clinicians.

\section{Conclusions}

When using goniometry for the assessment of hip range of motion and hand-held dynamometry for hip muscle strength in patients with hip osteoarthritis, reproducibility of individual measurements was generally poor between a pair of orthopaedists and a pair of chiropractors, indicating standardisation and rigorous training would be essential if this were to be improved. Both orthopaedists and chiropractors have a moderate ability to differentiate between hips without clinical osteoarthritis and hips assessed as having either mild or severe clinical osteoarthritis.

\section{Additional file}

Additional file 1: Examination protocol.

\section{Abbreviations}

OA: Osteoarthritis; ROM: Range of motion; Deg: Degrees; HHD: Hand-held dynamometer; N: Newton; ICC: Intraclass correlation coefficient; SD: Standard deviation; Cl: Confidence interval; LoA: Limits of agreement.

\section{Competing interests}

All authors declare that they have no competing interests.

\section{Authors' contributions}

EP, HWC, SO and $\mathrm{JH}$ contributed to the conception and design of the study. $E P, H W C, J \varnothing P$ and SO participated in the data collection. EP drafted the manuscript and performed the statistical analysis. EP, HWC, SO, W and $\mathrm{JH}$ participated in the interpretation of the data. All authors participated in the critical revision of the article and made important contributions to the content. All authors read and approved the final manuscript.

\section{Acknowledgements}

We would like to thank research secretary Jytte Johannesen for designing the recording forms, Suzanne Capell for proof reading the manuscript and project nurse Annie Gam-Pedersen for the organisation of patients and clinicians on the days of examination.

\section{Author details}

${ }^{1}$ Institute of Sports Science and Clinical Biomechanics, University of Southern Denmark, Odense, Denmark. ${ }^{2}$ Nordic Institute of Chiropractic and Clinical Biomechanics, Odense, Denmark. ${ }^{3}$ Department of Orthopaedic Surgery and Traumatology, Odense University Hospital, Odense, Denmark. ${ }^{4}$ Institute of Clinical Research, University of Southern Denmark, Odense, Denmark. ${ }^{5}$ Institute of Medical Biometry and Medical Informatics, University of Freiburg, Freiburg, Germany.

Received: 14 March 2012 Accepted: 30 November 2012

Published: 6 December 2012

\section{References}

1. Birrell F, Croft P, Cooper C, Hosie G, Macfarlane GJ, Silman A: Radiographic change is common in new presenters in primary care with hip pain. PCR Hip Study Group. Rheumatology (Oxford) 2000, 39:772-775.

2. Bierma-Zeinstra SM, Oster JD, Bernsen RM, Verhaar JA, Ginai AZ, Bohnen AM: Joint space narrowing and relationship with symptoms and signs in adults consulting for hip pain in primary care. J Rheumatol 2002, 29:1713-1718.

3. Cibere J: Do we need radiographs to diagnose osteoarthritis? Best Pract Res Clin Rheumatol 2006, 20:27-38.

4. Cibulka MT, White DM, Woehrle J, Harris-Hayes M, Enseki K, Fagerson TL, et al: Hip pain and mobility deficits-hip osteoarthritis: clinical practice guidelines linked to the international classification of functioning, disability, and health from the orthopaedic section of the American Physical Therapy Association. J Orthop Sports Phys Ther 2009, 39:A1-A25.

5. Arokoski MH, Haara M, Helminen HJ, Arokoski JP: Physical function in men with and without hip osteoarthritis. Arch Phys Med Rehabil 2004, 85:574-581.

6. Klassbo M, Harms-Ringdahl K, Larsson G: Examination of passive ROM and capsular patterns in the hip. Physiother Res Int 2003, 8:1-12. 
7. Pua YH, Wrigley TV, Cowan SM, Bennell KL: Intrarater test-retest reliability of hip range of motion and hip muscle strength measurements in persons with hip osteoarthritis. Arch Phys Med Rehabil 2008, 89:1146-1154.

8. Rydevik K, Fernandes L, Nordsletten L, Risberg MA: Functioning and disability in patients with hip osteoarthritis with mild to moderate pain. J Orthop Sports Phys Ther 2010, 40:616-624.

9. Rasch A, Bystrom AH, Dalen N, Berg HE: Reduced muscle radiological density, cross-sectional area, and strength of major hip and knee muscles in 22 patients with hip osteoarthritis. Acta Orthop 2007, 78:505-510

10. Pua YH, Wrigley TV, Collins M, Cowan SM, Bennell KL: Association of physical performance with muscle strength and hip range of motion in hip osteoarthritis. Arthritis Rheum 2009, 61:442-450.

11. Suetta C, Aagaard P, Magnusson SP, Andersen LL, Sipila S, Rosted A, et al: Muscle size, neuromuscular activation, and rapid force characteristics in elderly men and women: effects of unilateral long-term disuse due to hip-osteoarthritis. J Appl Physiol 2007, 102:942-948.

12. Birrell F, Croft P, Cooper C, Hosie G, Macfarlane G, Silman A: Predicting radiographic hip osteoarthritis from range of movement. Rheumatology (Oxford) 2001, 40:506-512.

13. Holla JF, Steultjens MP, van der Leeden M, Roorda LD, Bierma-Zeinstra SM, den Broeder AA, et al: Determinants of range of joint motion in patients with early symptomatic osteoarthritis of the hip and/or knee: an exploratory study in the CHECK cohort. Osteoarthr Cartil 2011, 19:411-419.

14. Holm I, Bolstad B, Lutken T, Ervik A, Rokkum M, Steen H: Reliability of goniometric measurements and visual estimates of hip ROM in patients with osteoarthrosis. Physiother Res Int 2000, 5:241-248.

15. Theiler R, Stucki G, Schutz R, Hofer H, Seifert B, Tyndall A, et al: Parametric and non-parametric measures in the assessment of knee and hip osteoarthritis: interobserver reliability and correlation with radiology. Osteoarthr Cartil 1996, 4:35-42.

16. Croft PR, Nahit ES, Macfarlane GJ, Silman AJ: Interobserver reliability in measuring flexion, internal rotation, and external rotation of the hip using a plurimeter. Ann Rheum Dis 1996, 55:320-323.

17. Cibere J, Thorne A, Bellamy N, Greidanus N, Chalmers A, Mahomed N, et al: Reliability of the hip examination in osteoarthritis: effect of standardization. Arthritis Rheum 2008, 59:373-381.

18. Rasch A, Dalen N, Berg HE: Test methods to detect hip and knee muscle weakness and gait disturbance in patients with hip osteoarthritis. Arch Phys Med Rehabil 2005, 86:2371-2376.

19. Sutlive TG, Lopez HP, Schnitker DE, Yawn SE, Halle RJ, Mansfield LT, et al: Development of a clinical prediction rule for diagnosing hip osteoarthritis in individuals with unilateral hip pain. $J$ Orthop Sports Phys Ther 2008, 38:542-550.

20. de Vet HC, Terwee CB, Knol DL, Bouter LM: When to use agreement versus reliability measures. J Clin Epidemiol 2006, 59:1033-1039.

21. Aalto TJ, Airaksinen O, Harkonen TM, Arokoski JP: Effect of passive stretch on reproducibility of hip range of motion measurements. Arch Phys Med Rehabil 2005, 86:549-557.

22. Prather H, Harris-Hayes M, Hunt DM, Steger-May K, Mathew V, Clohisy JC: Reliability and agreement of hip range of motion and provocative physical examination tests in asymptomatic volunteers. PM R 2010, 2:888-895.

23. Bierma-Zeinstra SM, Bohnen AM, Ramlal R, Ridderikhoff J, Verhaar JA, Prins A: Comparison between two devices for measuring hip joint motions. Clin Rehabil 1998, 12:497-505.

24. Bodenheimer T, Lorig K, Holman H, Grumbach K: Patient self-management of chronic disease in primary care. JAMA 2002, 288:2469-2475.

25. Poulsen E, Christensen HW, Roos EM, Vach W, Overgaard S, Hartvigsen J: Non-surgical treatment of hip osteoarthritis. Hip school, with or without the addition of manual therapy, in comparison to a minimal control intervention: Protocol for a three-armed randomized clinical trial. BMC Musculoskelet Disord 2011, 12:88.

26. Andrews AW, Thomas MW, Bohannon RW: Normative values for isometric muscle force measurements obtained with hand-held dynamometers. Phys Ther 1996, 76:248-259.

27. Bland JM, Altman DG: Statistical methods for assessing agreement between two methods of clinical measurement. Lancet 1986, 1:307-310.

28. Currier DP: Elements of Reseach in Physical Therapy. 3rd edition. Baltimore: Williams \& Wilkins; 1990.
29. Aaronson N, Alonso J, Burnam A, Lohr KN, Patrick DL, Perrin E, et al: Assessing health status and quality-of-life instruments: attributes and review criteria. Qual Life Res 2002, 11:193-205.

30. Weir JP: Quantifying test-retest reliability using the intraclass correlation coefficient and the SEM. J Strength Cond Res 2005, 19:231-240.

31. Landis JR, Koch GG: The measurement of observer agreement for categorical data. Biometrics 1977, 33:159-174.

32. Muller R, Buttner P: A critical discussion of intraclass correlation coefficients. Stat Med 1994, 13:2465-2476.

33. Kottner J, Audige L, Brorson S, Donner A, Gajewski BJ, Hrobjartsson A, et al: Guidelines for Reporting Reliability and Agreement Studies (GRRAS) were proposed. J Clin Epidemiol 2011, 64:96-106.

34. Wikholm JB, Bohannon RW: Hand-held Dynamometer Measurements: Tester Strength Makes a Difference. J Orthop Sports Phys Ther 1991 13:191-198.

35. Thorborg K, Bandholm T, Schick M, Jensen J, Holmich P: Hip strength assessment using handheld dynamometry is subject to intertester bias when testers are of different sex and strength. Scand J Med Sci Sports 2011, Oct 28: Epub ahead of print.

36. Arnold CM, Warkentin KD, Chilibeck PD, Magnus CR: The reliability and validity of handheld dynamometry for the measurement of lowerextremity muscle strength in older adults. J Strength Cond Res 2010, 24:815-824.

37. Lu YM, Lin JH, Hsiao SF, Liu MF, Chen SM, Lue YJ: The relative and absolute reliability of leg muscle strength testing by a handheld dynamometer. J Strength Cond Res 2011, 25:1065-1071.

38. Fulcher ML, Hanna CM, Raina EC: Reliability of handheld dynamometry in assessment of hip strength in adult male football players. I Sci Med Sport 2010, 13:80-84.

39. Cibere J, Bellamy N, Thorne A, Esdaile JM, McGorm KJ, Chalmers A, et al: Reliability of the knee examination in osteoarthritis: effect of standardization. Arthritis Rheum 2004, 50:458-468.

40. Brunse MH, Stochkendahl MJ, Vach W, Kongsted A, Poulsen E, Hartvigsen J, et al: Examination of musculoskeletal chest pain - an inter-observer reliability study. Man Ther 2010, 15:167-172.

41. Thorborg K, Petersen J, Magnusson SP, Holmich P: Clinical assessment of hip strength using a hand-held dynamometer is reliable. Scand J Med Sci Sports 2010, 20:493-501.

doi:10.1186/1471-2474-13-242

Cite this article as: Poulsen et al:: Reproducibility of range of motion and muscle strength measurements in patients with hip osteoarthritis an inter-rater study. BMC Musculoskeletal Disorders 2012 13:242.

\section{Submit your next manuscript to BioMed Central and take full advantage of:}

- Convenient online submission

- Thorough peer review

- No space constraints or color figure charges

- Immediate publication on acceptance

- Inclusion in PubMed, CAS, Scopus and Google Scholar

- Research which is freely available for redistribution 